\title{
Sutura Mendosal (Sutura Acessória): uma importante e quase desconhecida sutura craniana
}

Giuliano Roberto Goncalves 1, 2*, Rafael Cofiño de Sá 2, 3, Igor Esteban Umanzor Ordenes 1, 2, Bruno de Nardo Claudino 3, Rafael Cavalcante Barbosa 3, Leandro Henrique Grecco 4

${ }^{1}$ Departamento de Anatomia Humana, Faculdade de Medicina São Leopoldo Mandic, Araras, São Paulo/SP, Brasil. ${ }^{2}$ Departamento de Anatomia Humana, Centro Universitário UniMetrocamp, Campinas, São Paulo, Brasil.

${ }^{3}$ Faculdade de Medicina, Universidade São Francisco, Bragança Paulista, São Paulo/SP, Brasil.

${ }^{4}$ Departamento de Anatomia Humana, Faculdade São Leopoldo Mandic, Campinas, São Paulo/SP, Brasil.

*Autor correspondente: Giuliano Roberto Gonçalves. Av. Dona Renata, 71, Centro. CEP: 13606-134. Araras - São Paulo/SP. E-mail: giulianoanatomia@gmail.com

Aprovação do Comitê de Ética em Pesquisa com Seres Humanos: Aprovado pelo comitê de ética em Pesquisa do Centro Universitário UniMetrocamp sob o número de aprovação \#3.241.634.

Recebido em: Dez 26, 2021. Aceito em: Jan 14, 2021. Disponível online em: Jan 16, 2021.

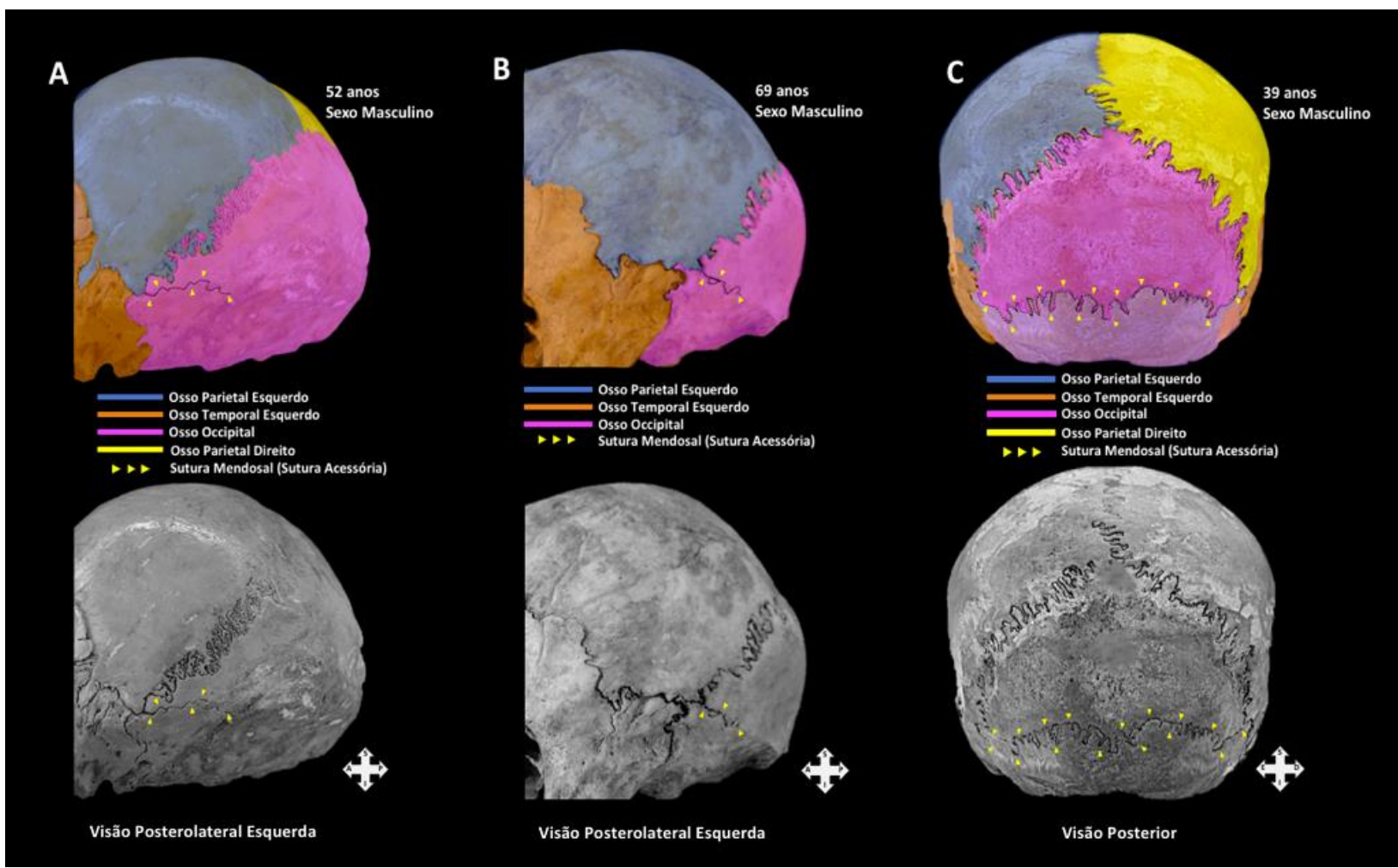

Figura 1. Sutura Mendosal (Sutura Acessória). A, crânio do sexo masculino, 52 anos, raça declarada como branca, Sutura Incompleta. B, crânio do sexo masculino, 69 anos, raça declarada como preta, Sutura Incompleta. C, crânio do sexo masculino, 39 anos, raça declarada como branca, Sutura Completa. 
Há uma escassez de informações na literatura sobre a Sutura Mendosal (Sutura Acessória). Além disso, os relatos do fechamento (sinostose) dessa suposta sutura da infância são variáveis $[1,2]$. O osso occipital é funcionalmente e ontogenética único quando comparado aos outros ossos do crânio em humanos e também com outros mamíferos $[1,4]$. O osso occipital se desenvolve a partir de seis centros de ossificação; qualquer deformidade no processo de ossificação dará origem a Sutura Mendosal (Sutura Acessória) e/ou condições atípicas como plagiocefalia posterior [3, 5]. Além da escassez de literatura sobre a sutura mendosal, seu relato na população brasileira é quase desconhecido $[1,3,5]$.

$\mathrm{O}$ objetivo desse estudo foi verificar a presença de Suturas Mendosais em crânios humanos secos adultos numa coleção óssea do estado de São Paulo. Trata-se de um estudo anatômico observacional numa coleção óssea completa e identificada, pertencente ao Laboratório de Anatomia Humana do Centro Universitário UniMetrocamp. Foram analisados 53 crânios humanos secos adultos, que fazem parte da coleção óssea. Em cada crânio foi examinado a presença ou não da Sutura Mendosal. Foram excluídos da amostra crânios que possuíam algum tipo de dano na região de análise.
Os registros fotográficos foram realizados por câmera digital de alta resolução. Dos 53 crânios analisados, a presença de Sutura Mendosal ocorreu em 03 crânios. As figuras $1 \mathrm{~A}$ e 1B apresentam a presença da Sutura Mendosal em crânios de dois indivíduos do sexo masculino de raça declarada branca, com idade de 52 e 69 anos, respectivamente, de forma incompleta lateralmente (lado esquerdo) do crânio, não possuindo simetrias bilaterais.

A figura $1 \mathrm{C}$ representa a presença da Sutura Mendosal completa em crânio de indivíduo do sexo masculino de raça declarada como preta, com idade de 39 anos, possuindo simetria bilateral da sutura. As definições de sexo, idade e etnias foram estabelecidas pelo Serviço de Verificação de Óbito (SVO) do Estado de São Paulo.

Este tema manifesta grande relevância pois a diferenciação entre fraturas e suturas acessórias é feita pelo conhecimento da anatomia normal e do tempo de fechamento das suturas (sinostoses), especialmente na região occipital [1, 5]. Por se tratar de crânios adultos, esta sutura chamada Mendosal é uma sutura persistente, tal como, a Sutura Metópica encontrada no osso frontal $[2,5]$.

Acreditamos que esses dados serão úteis para médicos radiologistas, neurocirurgiões e intensivistas, a fim de evitar quaisquer interpretações errôneas 
da Sutura Mendosal associando-a com fraturas cranianas, bem como, fornecer dados anatômicos para profissionais das ciências forenses.

\section{Referências}

[1] Gayretli O, Gurses IA, Kale A, Aksu F, Ozturk A, Bayraktar B, Sahinoglu K. The mendosal suture. Br J Neurosurg. 2011 Dec;25(6):730-3. doi: 10.3109/02688697.2010.544789.

[2] Nayak SR, Krishnamurthy A, Madhan Kumar SJ, Prabhu LV, Jiji PJ, Pai MM, Kumar A, Avadhani R. The mendosal suture of the occipital bone: occurrence in Indian population, embryology and clinical significance. Surg Radiol Anat. 2007.Jun;29(4):329-32. doi:10.1007/s00276-007-0216-2.

[3] Ruparelia J, Patidar R, Gosal JS, Garg M, Bhaskar S, Jha DK. An Aberrant Line on CT Head: The Mendosal Suture. J Neurosci Rural Pract. 2020;11(3):502-503. doi:10.1055/s-0040-1713304.

[4] Gurses IA, Esenkaya A, Gayretli O, Kale A, Ozturk A, Tekes A. A new anatomic trait for identifying the mendosal suture in young children: the mendosal-lambdoidal angle. Surg Radiol Anat. 2016 Apr;38(3):321-5. doi: 10.1007/s00276-015-1556-y.

[5] Smith LGF, Governale LS, Sribnick EA. A Persistent Mendosal Suture Presenting With Bathrocephaly. Pediatr Neurol. 2017 Aug;73:110. doi: 10.1016/j.pediatrneurol.2017.04.019.
Conflitos de interesses: Todos os autores declaram não haver quaisquer conflitos de interesse.

Agradecimentos: Agradecemos ao Centro Universitário UniMetrocamp pelo acesso ao Laboratório e Acervo Ossuário.

Financiamentos: Não se aplica.

Como citar este artigo: Gonçalves GR, Sá RC, Ordenes IEU, Claudino BN, Barbosa RC, Grecco LH. Sutura Mendosal (Sutura Acessória): uma importante e quase desconhecida sutura craniana. Brazilian Journal of Case Reports. 2022 Jan-Mar;02(1):29-31. 